\title{
A New Approach for a Class of Optimal Control Problems of Volterra Integral Equations
}

\author{
Mohammad Hadi Noori Skandari ${ }^{1}$, Hamid Reza Erfanian ${ }^{2}$, Ali Vahidian Kamyad ${ }^{1}$ \\ ${ }^{1}$ Department of Applied Mathematics, School of Mathematical Sciences, Ferdowsi \\ University of Mashhad, Mashhad, Iran \\ ${ }^{2}$ Department of Mathematics \& Statistics, University of Science and Culture, Tehran, Iran \\ E-mail: hadinoori344@yahoo.com,Erfanian@usc.ac.ir,avkamyad@yahoo.com \\ Received January 30, 2011; revised March 23, 2011; accepted May 5, 2011
}

\begin{abstract}
In this paper, we propose a new approach for a class of optimal control problems governed by Volterra integral equations which is based on linear combination property of intervals. We convert the nonlinear terms in constraints of problem to the corresponding linear terms. Discretization method is also applied to convert the new problems to the discrete-time problem. In addition, some numerical examples are presented to illustrate the effectiveness of the proposed approach.
\end{abstract}

Keywords: Volterra Integral Equations, Optimal Control, Linear Programming

\section{Introduction}

Consider the following optimal control problem governed by Volterra integral equation $(\mathrm{OCV})$ :

$$
\begin{gathered}
\text { minimize } G(y(T))+\int_{0}^{T} F(t, y(t), u(t)) \mathrm{d} t \\
\text { subject to } y(t)=p(t)+\int_{0}^{t} K(t, s, y(s), u(s)) \mathrm{d} s, \\
0 \leq t \leq T
\end{gathered}
$$

where $y($.$) and u($.$) are the state and control func-$ tions respectively on $[0, T]$. The integral Equation (2) is applied in a natural way in the study of economic problems, population dynamics and etc., see for instance Hritonenko and Yatsenco [1], and Kamien and Schwartz [2]. The OCV problem (1)-(2) has been studied by many authors, including Neustadt [3-5], Bakke [6], Carlson [7], Vinokurov [8], Medhim [9], Schmidt [10-13], Wolfansdorf [14], Elnagar, Kazemi and Kim [15], Pan and Teo [16], Angell [17,18], Belbas [19,20], Carlier and Tahraoui [21], and Burnap, and Kazemi [22]. The method usually employed for OCV problem (1)-(2) is method of necessary conditions of the type of Pontryagin maximum principle. In the Recent works, Vega [23] gives the necessary condition for optimal terminal time of OCV problem (1)-(2) and verifies the terminal time $T$ and final state $y(T)$ by conditions $\varphi(T, y(T))=0$ and $\psi(T, y(T)) \leq 0$. Bonnens and Vega [24] discuss problem (1)-(2) with running state on the initial and final states. Also, Belbas
[25] applied the ideas of dynamic programming to OCV problem (1)-(2).

In this paper, we are interested to solving the following class of the OCV problem (1)-(2) which we called it COCV problem:

$$
\operatorname{minimize} \int_{0}^{T} c(t) y(t) \mathrm{d} t
$$

$$
\begin{gathered}
\text { subject to } y(t)=p(t)+\int_{0}^{t}[f(s, u(s))+\mathrm{d}(t, s) y(s)] \mathrm{d} s, \\
0 \leq t \leq T
\end{gathered}
$$

where function $f(.,$.$) is a continuous function. A con-$ trolled Volterra integral equation similar to equation (4) is discussed in [16]. We suppose that $u(t) \in U$, $t \in[0, T]$ where $U$ a compact and connected set. In addition, we let the final state $y(T)$ is a given known number. Here, the linear combination property of intervals is used to convert nonlinear controlled Volterra integral Equation (4) to the equivalent linear equation. The new optimal control problem with this linear Volterra integral equation is transformed to a discrete-time problem that could be solved by linear programming methods. This paper organized as follows. Section 2, transforms the nonlinear function $f(.,$.$) to a corresponding function$ that is linear respect to a new control function. Section 3, converts the new problem to the discrete-time problem via discretization. In Section 4, numerical examples are presented to illustrated effectivness of this approach. 
Finally, the conclusion of this paper is given in Section 5.

\section{Metamorphosis of the COCV Problem}

In this section, COCV problem (1) is transformed to the new equivalent problem. First, we state and prove the following two theorems:

Theorem 2.1: Let $f(.,$.$) be a continuous function on$ $[0, T] \times U$ where $U$ is a compact and connected subset of $\mathbb{R}^{m}$, then for any arbitrary (but fixed) $s \in[0, T]$ the set $\{f(s, u): u \in U\}$ is a closed interval in $\mathbb{R}$.

Proof: Assume that $s \in[0, T]$ be given. Let $\phi(u)=$ $f(s, u)$. Obviously, $\phi($.$) is a continuous function on U$. Since, continuous function preserve compactness and connectedness, the set $\{\varphi(u): u \in U\}$ is compact and connected. Therefore, $\{\varphi(u): u \in U\}$ is a closed interval in $\mathbb{R} . \square$

For any $s \in[0, T]$, we may suppose the lower and upper bounds of interval $\{F(s, u): u \in U\}$ are $g(s)$ and $w(s)$ respectively. Thus we have:

$$
g(s) \leq f(s, u) \leq w(s), \quad s \in[0, T]
$$

In other words

$$
\begin{aligned}
& g(s)=\min _{u}\{f(s, u): u \in U\}, \quad s \in[0, T] \\
& w(s)=\max _{u}\{f(s, u): u \in U\}, \quad s \in[0, T]
\end{aligned}
$$

Theorem 2.2: Let functions $g($.$) and w($.$) be de-$ fined by relations (6) and (7). Then they are uniformly continuous on $[0, T]$.

Proof: We will show that $g($.$) is a uniformly con-$ tinuous function. It is sufficient that we show that for any given $\varepsilon>0$, there exists $\delta>0$ such that if $s_{1} \in N_{\delta}\left(s_{2}\right)$ then $\left|g\left(s_{1}\right)-g\left(s_{2}\right)\right|<\varepsilon$ where $N_{\delta}(z)$ is a $\delta$-neighborhood of $Z$. Since, any continuous function on compact set is a uniformly continuous. The function $f(.,$.$) on compact set [0, T] \times U$ is a uniformly continuous, i.e. for any $\varepsilon>0$ there exists $\delta>0$, such that if $\left(s_{1}, u\right) \in N_{\delta}\left(s_{2}, u\right)$ then $\left|f\left(s_{1}, u\right)-f\left(s_{2}, u\right)\right|<\varepsilon$. Thus $f\left(s_{1}, u\right)<f\left(s_{2}, u\right)+\varepsilon$. In addition, by (5), $g\left(s_{1}\right) \leq f\left(s_{1}, u\right)$ and so $g\left(s_{1}\right) \leq$ $f\left(s_{2}, u\right)+\varepsilon$. Now, by taking infimum on the right hand side of the last inequality $g\left(s_{1}\right) \leq g\left(s_{2}\right)+\varepsilon$. By a similar procedure, we have $g\left(s_{2}\right)-g\left(s_{1}\right) \leq \varepsilon$. Thus $\left|g\left(s_{1}\right)-g\left(s_{2}\right)\right| \leq \varepsilon$. The proof of uniformly continuity of $w($.$) is similar. \square$

By linear combination property of intervals and relation (5), we have for any $s \in[0, T]$ :

$$
f(s, u(s))=(w(s)-g(s)) \lambda(s)+g(s), \lambda(s) \in[0,1]
$$

Thus, we transform COCV problem (3)-(4) by relation
(6) as the following continuous-time problem:

$$
\text { minimize } \int_{0}^{T} c(t) y(t) \mathrm{d} t
$$

$$
\begin{gathered}
\text { subject to } y(t)=q(t)+\int_{0}^{t}[h(s) \lambda(s)+d(t, s) y(s)] \mathrm{d} s, \\
0 \leq \lambda(t) \leq 1, t \in[0, T]
\end{gathered}
$$

where $q(t)=p(t)+\int_{0}^{t} g(s) \mathrm{d} s$ and $h(t)=w(t)-g(t)$ for any $t \in[0, T]$. Note that in the new problem (9), which is a optimal control of linear Volterra integral equation, $\lambda($.$) is the new control function. Next section,$ converts the problem (9) to the corresponding linear programming problem.

\section{Discrete-Time Problem}

Now, discretization method enables us transforming continuous problem (9) to the corresponding discrete form. Consider equidistance points $s_{0}=0<s_{1}<s_{2}<\cdots<s_{N}=T$ of interval $[0, T]$ which defined as

$$
s_{j}=\frac{T}{N} j, j=0,1, \cdots, N
$$

where $N$ is a given big number. Also, we set $t_{j}=s_{j}$ for $j=0,1, \cdots, N$. By trapezoidal approximation in numerical integration, problem (9) is converted to the following discrete-time problem:

$$
\operatorname{minimize}\left(\frac{T}{2 N} c_{0} y_{0}+\frac{T}{N} \sum_{j=1}^{N-1} c_{j} y_{j}+\frac{T}{2 N} c_{N} y_{N}\right)
$$

subject to

$$
\begin{aligned}
& \left(1-\frac{T}{2 j} d_{j j}\right) y_{j}-\frac{T}{2 j} h_{0} \lambda_{0}-\frac{T}{j} \sum_{i=1}^{j-1}\left(h_{0} \lambda_{i}+d_{j i} y_{i}\right)-\frac{T}{2 j} h_{j} \lambda_{j} \\
& =q_{j}+\frac{T}{2 j} d_{j 0} y_{0}, \\
& 0 \leq \lambda_{j} \leq 1, y_{0}=q_{0}, y_{N}=\eta j=0,1,2, \cdots, N
\end{aligned}
$$

where

$y_{j}=y\left(t_{j}\right), \quad h_{j}=h\left(s_{j}\right), \quad d_{i j}=d\left(t_{i}, s_{j}\right), \quad c_{j}=c\left(s_{j}\right)$, $\lambda_{j}=\lambda\left(t_{j}\right)$ and $q_{j}=q\left(t_{j}\right)$ for all $i, j=0,1,2, \cdots, N$. In problem (10), final state is $\eta$ that is a known number. By solving problem (10), which is a linear programming problem, we are able to obtain the optimal solution $\lambda_{j}^{*}$ and $y_{j}^{*}$ for all $j=0,1,2, \cdots, N$. Note that, for evaluating optimal control variable $u^{*}($.$) , we must use the fol-$ lowing equation:

$$
f\left(s, u^{*}\right)=h(s) \lambda^{*}+g(s) .
$$

\section{Numerical Examples}

Here, we use our approach to obtain approximate optimal 
solution of the following two COCV problems by solving linear programming (LP) problem (10) via simplex method [26] in MATLAB software.

Example 4.1: Consider the following COCV problem: $\operatorname{minimize} \int_{0}^{1} \cos (3 \pi t) y(t) \mathrm{d} t$

subject to

$$
\begin{aligned}
& y(t)=\sin (3 \pi t)+\int_{0}^{t}\left[\sin \left(\frac{\pi}{4} u(s)+s\right)\right. \\
& +\cos (3(t-s) \pi) y(s)] \mathrm{d} s \\
& 0 \leq u(t) \leq 0.5, t \in[0,1] \\
& y(1)=1 \text {. }
\end{aligned}
$$

Here, $f(s, u)=\sin \left(\frac{\pi}{4} u+s\right), d(t, s)=\cos 3(t-s) \pi$ $c(t)=\cos (3 \pi t)$ and $p(t)=\sin (3 \pi t)$ for all $t \in[0,1]$, $s \in[0,1]$ and $u \in[0,0.5]$. Thus by (6), (7)

$$
\begin{gathered}
g(s)=\min _{u \in[0,0.5]}\left\{\sin \left(\frac{\pi}{4} u+s\right)\right\}=\sin (s), s \in[0,1] \\
w(s)=\max _{u \in[0,0.5]}\left\{\sin \left(\frac{\pi}{4} u+s\right)\right\}=\sin \left(\frac{\pi}{8}+s\right), s \in[0,1] .
\end{gathered}
$$

hence

$$
\begin{gathered}
q(t)=p(t)+\int_{0}^{t} g(s) \mathrm{d} s=\sin (3 \pi t)-\cos (t)+1, t \in[0,1] \\
h(s)=w(s)-g(s)=\sin \left(\frac{\pi}{8}+s\right)-\sin (s), s \in[0,1]
\end{gathered}
$$

Assume that $N=100$ and $s_{j}=\frac{j}{100}$ for all $j=0,1$, $2, \cdots, 100$. The optimal solutions $y_{j}^{*}$ and $\lambda_{j}^{*}, j=0,1$, $2, \cdots, 100$ of problem (12) is obtained by solving problem (10) which is illustrated in Figures 1 and 2 respectively. Here, the value of optimal solution of objective function is -0.470 . The corresponding Equation (11) for this example is

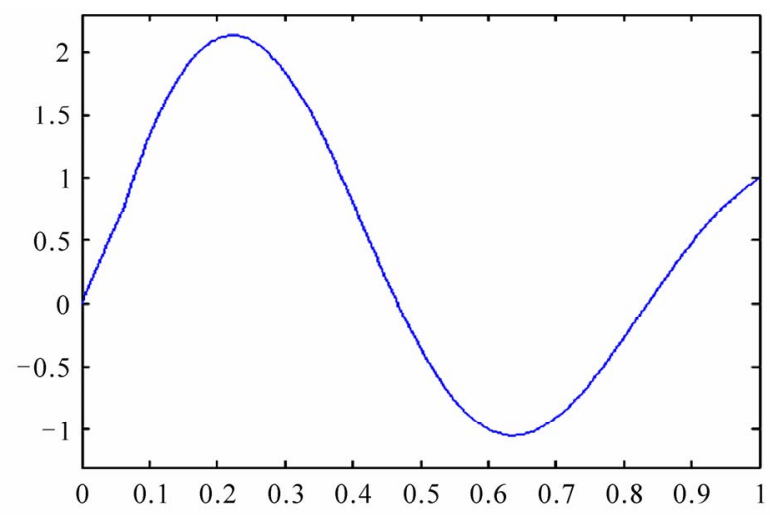

Figure 1. Optimal trajectory $y^{*}($.$) of Ex.12.$

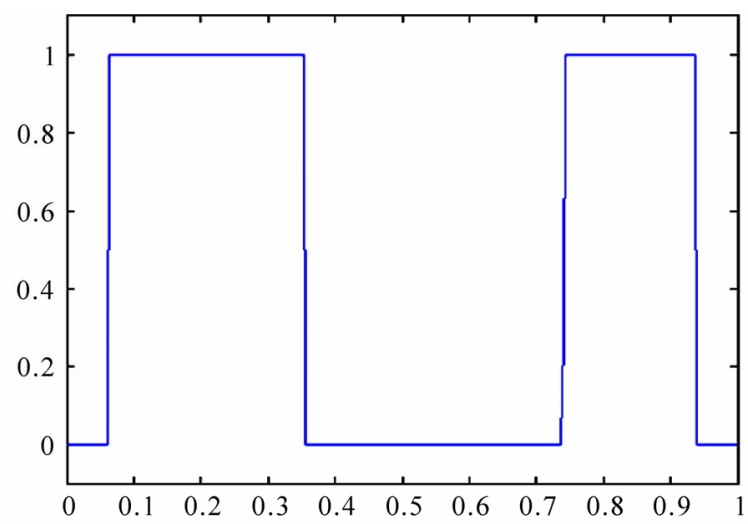

Figure 2. Corresponding optimal control $\lambda^{*}($.$) of Ex.12.$

$$
\sin \left(\left(\frac{\pi}{4}\right) u_{j}^{*}+s_{j}\right)=h\left(s_{j}\right) \lambda_{j}^{*}+g\left(s_{j}\right), j=0,1,2, \cdots, 100
$$

therefore

$$
u_{j}^{*}=\frac{4}{\pi}\left(\sin ^{-1}\left(h\left(s_{j}\right) \lambda_{j}^{*}+g\left(s_{j}\right)\right)-s_{j}\right), j=0,1,2, \cdots, 100
$$

The optimal control $u_{j}^{*}, \quad j=0,1,2, \cdots, 100$ of this example is showed in Figure 3.

Example 4.2: Consider the following COCV problem:

$$
\operatorname{minimize} \int_{0}^{1}(0.5-t) y(t) \mathrm{d} t
$$

subject to $y(t)=t-\int_{0}^{t}\left(3 \ln \left(u^{3}(s)+s+2\right)+t e^{t s} y(s)\right) \mathrm{d} s$

$$
\begin{aligned}
& 0 \leq u(t) \leq 1, t \in[0,1] \\
& y(1)=-1 .
\end{aligned}
$$

Here $\left.f(s, u)=-3 \ln \left(u^{3}(s)+s+2\right), d(t, s)\right)=-t e^{t s}$, $c(t)=0.5-t$ and $p(t)=t$ for all $t \in[0,1], s \in[0,1]$ and $u \in[0,1]$. In this example for all $s \in[0,1]$

$$
\begin{gathered}
g(s)=\min _{u \in[0,1]}\left\{-3 \ln \left(u^{3}(s)+s+2\right)\right\}=-3 \ln (s+3), \\
w(s)=\max _{u \in[0,1]}\left\{-3 \ln \left(u^{3}(s)+s+2\right)\right\}=-3 \ln (s+2), \\
h(s)=w(s)-g(s)=3(\ln (s+3)-\ln (s+2)) .
\end{gathered}
$$

Moreover for all $t \in[0,1]$

$$
\begin{aligned}
q(t) & =p(t)+\int_{0}^{t} g(s) \mathrm{d} s \\
& =t-3((t+3) \ln (t+3)-(t+3)-3 \log (3)+3),
\end{aligned}
$$

Let $N=100$ and $t_{j}=\frac{j}{100}$ for all $j=0,1,2, \cdots, 100$. We obtain the optimal solution $y_{j}^{*}$ and $\lambda_{j}^{*}, j=0,1$, $2, \cdots, 100$ of problem (13) by solving problem (10) which is illustrated in Figures $\mathbf{4}$ and $\mathbf{5}$ respectively. In 


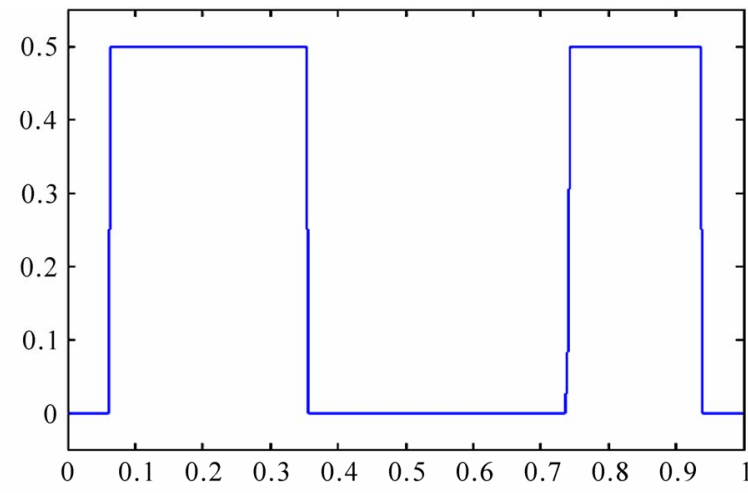

Figure 3. Optimal control $u^{*}($.$) of Ex.12.$

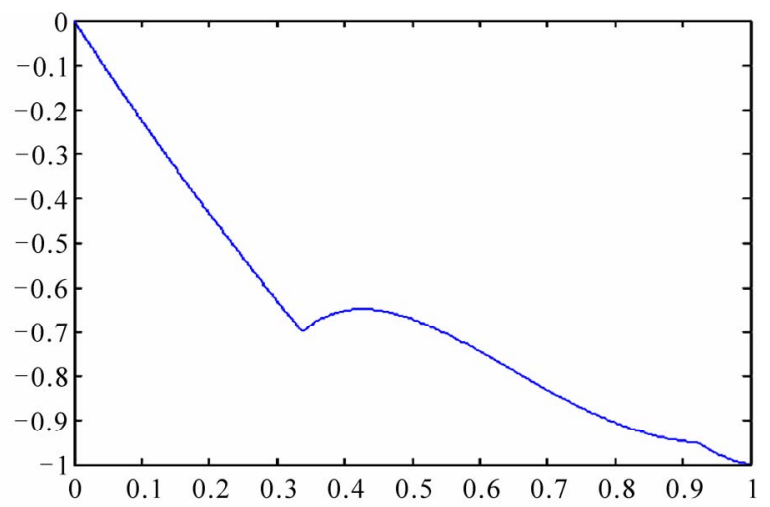

Figure 4. Optimal trajectory $y^{*}($.$) of Ex.13.$

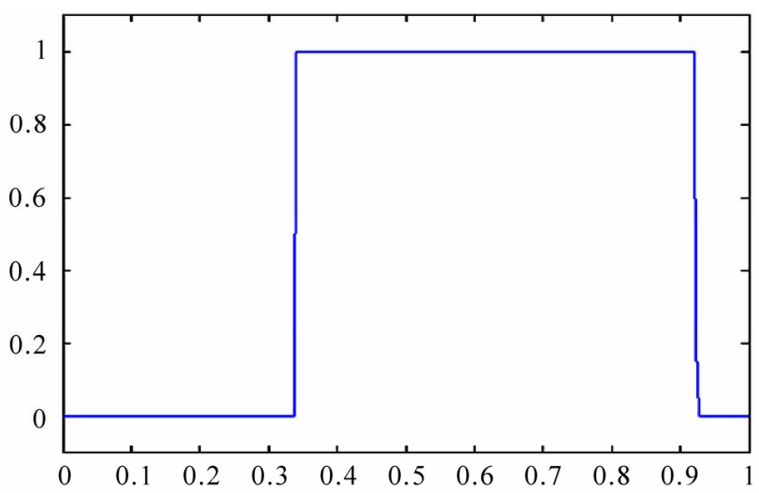

Figure 5. Corresponding optimal control $\lambda^{*}($.$) of Ex.13.$

addition, by (11) the corresponding $u^{*}($.$) for this ex-$ ample is

$$
u_{j}^{*}=\left(e^{-\left(h\left(s_{j}\right) \lambda_{j}^{*}+g\left(s_{j}\right)\right) / 3}-s_{j}-2\right)^{1 / 3}, j=0,1,2, \cdots, 100
$$

The optimal control $u_{j}^{*}, j=0,1,2, \cdots, 100$ of problem (10) is showed in Figure 6. Here, the value of optimal solution of objective function is 0.071 .

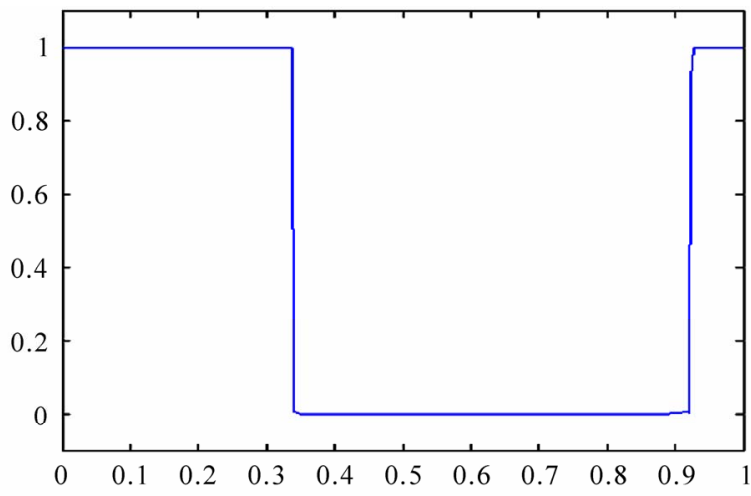

Figure 6. Optimal control $u^{*}($.$) of Ex.13.$

\section{Conclusions}

In this paper, we posed a different approach for a class of nonlinear optimal control problem including Volterra integral equations. In our approach, the linear combination property of intervals is used to obtain the new corresponding problem which is a linear problem. The new problem can be converted to a LP problem by discretezation method. Finally, we obtain an approximate solution for the main problem. In next works, we are going to use our approach for subclasses of problem (1)-(2) which Volterra integral equation is similar to Equation (4), but objective functional is quadratic or nonlinear with respect to state variables.

\section{References}

[1] N. Hritonenko and Y. Yatsenko, "Mathematical Modeling in Economics, Ecology, and the Environment," Kluwer Academic Publishers, Dordrecht, 1999.

[2] M. I. Kamien and N. L. Schwartz, "Dynamic Optimization. The Calculus of Variations and Optimal Control in Economics and Management," North-Holland Publishing Co., Amsterdam, 1991.

[3] L. W. Neustadt, "Optimization," Princeton University Press, Princeton, 1976.

[4] L. W. Neustadt, "Optimization: A Theory of Necessary Conditions," Princeton University Press, Princeton, New Jersey, 1976.

[5] L. W. Neustadt and J. Warga, "Comments on the Paper 'Optimal Control of Processes Described by Integral Equations, I' by V. R. Vinokurov," SIAM Journal on Control and Optimization, Vol. 8, 1970, p. 572. doi:10.1137/0308041

[6] V. L. Bakke, "A Maximum Principle for an Optimal Control Problem with Integral Constraints," Journal of Optimization Theory and Applications, Vol. 13, No. 1, 1974, pp. 32- 55. doi:10.1007/BF00935608

[7] D. A. Carlson, "An Elementary Proof of the Maximum Principle for Optimal Control Problems Governed by a 
Volterra Integral Equation," Journal of Optimization Theory and Applications, Vol. 54, No. 1, 1987, pp. 43-61. doi:10.1007/BF00940404

[8] V. R. Vinokurov, "Optimal Control of Processes Described by Integral Equations, Parts I, II, and III," SIAM Journal of Control, Vol. 7, 1996, pp. 324-355. doi: $10.1137 / 0307022$

[9] N. G. Medhin, "Optimal Processes Governed by Integral Equations," Journal of Mathematical Analysis and Applications, Vol. 120, No. 1, 1986, pp. 1-12. doi:10.1016/0022-247X(86)90199-X

[10] W. H. Schmidt, "Notwendige Optimalitaetsbedingungen fuer Prozesse mit Zeitvariablen Integralgleichungen in Banach-Raeumen," Zeschrift fur Angewandte Mathematik und Mechanik, Vol. 60, 1980, pp. 595-608. doi:10.1002/zamm.19800601107

[11] W. H. Schmidt, "Durch Integralgleichungen Beschriebene Optimale Prozesse mit Nebenbedingungen in Banachraeumen Notwendige Optimalitaetsbendingungen, Zeschrift fur Angewandte Mathematik und Mechanik, Vol. 62, 1982, pp. 66-75.

[12] W. H. Schmidt, "Volterra Integral Processes with State Constraints," Scottish Association for Marine Science, Vol. 9, 1992, pp. 213-224.

[13] W. H. Schmidt, "Durch Integralgleichungen Beschrienbene Optimale Prozesse mit Nebenbedingungen in Banachraumen - Notwendige Optimalita Tsbedingungen," Zeschrift fur Angewandte Mathematik und Mechanik, Vol. 62, 1982, pp. 65-75.

[14] L. Wolfersdorf, "Optimale Steuerung bei Hammerstein schen Integralgleichungen mit Schwach Singulaeren Kernen," Math. Oper. Statist., Vol. 6, 1975, pp. 609-626.

[15] G. N. Elnagar, M. A. Kazemi and H. Kim, "Necessary and Sufficient Optimality Condittions for Control Systems Described by Integral Equations with Delay," Journal of the Korean Mathematical Society, Vol. 37, No. 4, 2000, pp. 625-643.

[16] L. P. Pan and K. L. Teo, "Near-Optimal Controls of a Class of Volterra Integral Systems1," Journal of Optimization Theory and Applications, Vol. 101, No. 2, 1999, pp. 355-373. doi:10.1023/A:1021741627449
[17] T. S. Angell, "Existence of Optimal Control without Convexity and a Bangbang Theorem for Linear Volterra Equations," Journal of Optimization Theory and Applications, Vol. 19, No. 1, 1976, pp. 63-79. doi:10.1007/BF00934052

[18] T. S. Angell, "On the Optimal Control of Systems Governed by Nonlinear Volterra Equations," Journal of Optimization Theory and Applications, Vol. 19, No. 1, 1976, pp. 29-45. doi:10.1007/BF00934050

[19] S. A. Belbas, "Iterative Schemes for Optimal Control of Volterra Integral Equations," Nonlinear Analysis, Vol. 37, No. 1, 1999, pp. 57-79. doi:10.1016/S0362-546X(98)00144-8

[20] S. A. Belbas, "A New Method for Optimal Control of Volterra Integral Equations," Applied Mathematics and Computation, Vol. 189, No. 1, 2007, pp. 1902-1915. doi:10.1016/j.amc.2006.12.077

[21] G. Carlier and R. Tahraoui, "On Some Optimal Control Problems Governed by a State Equation with Memory," ESAIM: Control, Optimisation and Calculus of Variations, Vol. 14, No. 4, 2008, pp. 725-743. doi:10.1051/cocv:2008005

[22] C. Burnap and M. Kazemi, "Optimal Control of a System Governed by Nonlinear Volterra Integral Equations with Delay," IMA Journal of Mathematical Control and Information, Vol. 16, No. 1, 1999, pp. 73-89. doi:10.1093/imamci/16.1.73

[23] C. de la Vega, "Necessary Conditions for Optimal Terminal Time Control Problems Governed by a Volterra Integral Equation," Journal of Optimization Theory and Applications, Vol. 130, No. 1, 2006, pp. 79-93. doi:10.1007/s10957-006-9087-7

[24] J. F. Bonnens and C. de la Vega, "Optimal Control of Stated Constrained Integral Equations," Institut national de Recherche en Informatiqeue et en Automatique, 2010.

[25] S. A. Belbas, "A Reduction Method for Optimal Control of Volterra Integral Equations," Applied Mathematics and Computation, Vol. 197, No. 2, 2008, pp. 880-890. doi:10.1016/j.amc.2007.08.093

[26] D. Luenberger, "Linear and Nonlinear Programming," Addison-Wesley, New York, 1984. 